\title{
Anisotropic off-normal incidence optical reflection from GaP (110) surfaces
}

\author{
C M J Wijers and G P M Poppe, Faculty of Applied Physics, Twente University of Technology, P.O. Box \\ 217, 7500 AE Enschede, The Netherlands
}

This article contains a theoretical study for off-normal incidence surface induced optical anisotropy (SIOA). The discrete dipole approximation was used to calculate the off-normal incidence optical response of slabs. By means of the two slab approach those results were converted to semi-infinite reflectivities. The calculated ellipsometric parameter $\delta \Delta$ shows large variations near the Brewster angle, but only the p-polarized reflection has a clearly increased SIOA sensitivity. So experimentally a straightforward determination of $\Delta \mathrm{R}_{\mathrm{p}}$ should be preferred. Advantages have to be sought in the optical observation of surface state related phenomena at subbandgap conditions.

\section{Introduction}

The experimental observation of surface induced optical anisotropy (SIOA) shown by 110 surfaces of cubic materials has reopened the discussion about the theoretical interpretation of optical reflection ${ }^{1-4}$. At first improvement was sought in a modified continuum approach ${ }^{5.6}$. With the aim to incorporate local field effects Mochán and Barrera added discrete elements to the continuum approach ${ }^{7}$. Wijers and Emmett ${ }^{1}$ started a discrete dipole description of SIOA. Using results obtained before by Ewald $^{8}$ and Litzman and Rózsa ${ }^{9.10}$, Wijers and Del Sole ${ }^{2}$ calculated the SIOA of a GaP 110 surface by means of the two slab approach for perpendicular incidence. The hybrid discretecontinuum character of this method has been overcome by Poppe and Wijers ${ }^{11}$ using the asymptotic continuation approach. That even the hybrid technique used in ref 2 offers substantial progress will be shown in this paper. It offers the first calculation of SIOA for off-normal incidence. Because the required full incorporation of retardation has been included already from the very beginning, it suffices to describe only the technical modifications necessary to turn the method into a feasible approach. Despite the complexity of the mathematics, the transparency of the solution from the physics point of view remains due to the usage of only four starting points ${ }^{1,2}$.

\section{Optical response of slabs}

2.1. Description of the configuration. The crystalline bulk is located in the upper halfspace and the electromagnetic beam impinges from the lower halfspace ${ }^{9,10}$. For GaP 110 the interlayer spacing $\mathbf{d}$ becomes:

$\mathbf{d}=(\sqrt{2} / 4,1 / 2, \sqrt{2} / 4) a^{\mathrm{c}}$

$a^{\mathrm{c}}$ represents the lattice constant. For off normal incidence we define $k_{\|}$, the component of $\mathbf{k}$ along the surface, as:

$\boldsymbol{k}=\left(k_{x}, k_{y}, k_{z}\right)=\left(\mathbf{k}_{\|}, k_{z}\right)$

$\mathbf{k}$ represents the wave vector of the beam. $\mathbf{k}_{\|}$being different from zero, results in an increased role of retardation for off normal incidence.
2.2. Dipole theory for slabs. The basic starting points of the description, e.g. using Hertz potentials and the principles of induction, superposition and parallel translational symmetry, have been treated in ref 2 . After combination they yield the general equations of dipole theory for slabs $\left(\alpha_{0}=4 \pi \varepsilon_{0} a^{3}, a=\right.$ $\left.a^{\mathrm{c}} / \sqrt{2}\right)$ :

$$
\begin{aligned}
\mathbf{p}_{i} & =\overleftrightarrow{\alpha}_{i}\left[\mathbf{E}_{\mathrm{EXT}, i}+\alpha_{0}^{-1} \sum_{j} \overleftrightarrow{\mathbf{f}_{i j}} \cdot \mathbf{p}_{j}\right] \\
\overleftrightarrow{\mathbf{f}}_{i j} & =\left.a^{3}\left[\nabla \nabla^{T}+k^{2} \overleftrightarrow{\mathbf{1}}\right] S_{j}(\mathbf{r}, \mathbf{k})\right|_{\mathbf{r}=r i} \\
S_{j}(\mathbf{r}, \mathbf{k}) & =\sum_{q r}^{\left({ }^{\prime}\right)} \exp \left(i \mathbf{k} \cdot \mathbf{s}_{q r}\right) \exp \left(i k\left|\mathbf{r}-\mathbf{r}_{j, q r}\right|\right) /\left|\mathbf{r}-\mathbf{r}_{j, q r}\right|
\end{aligned}
$$

The $\mathbf{p}_{i}$ 's, used in (3), refer to the characteristic dipoles of plane $i$, the $\mathbf{p}_{i, 00}$ as described already in ref 2 . The description (3-5) is fully dyadic in three dimensions. Like for normal incidence, also here it is not necessary to use the full $3 d$ approach. We start from the equations derived in ref 12 :

$$
\begin{aligned}
\overleftrightarrow{\mathbf{f}}_{i j} & =\frac{2 \pi i a^{3}}{\left|\mathbf{s}_{1} \times \mathbf{s}_{2}\right|} \sum_{p q}^{\infty}\left(k^{2} \stackrel{\leftrightarrow}{\mathbf{1}}-\mathbf{k}_{p q} \mathbf{l} \stackrel{\oplus}{p q}^{\mathcal{P}}\right) \frac{\exp \left(i \mathbf{k}_{p q} \cdot\left(\mathbf{r}_{i}-\mathbf{r}_{j}\right)\right)}{\kappa_{p q}} \\
\mathbf{k}_{p q} & =\left(\mathbf{k}_{\|}^{p q}, \mathbf{k}_{\perp}^{p q}\right)=\left(\mathbf{k}_{\|}+\mathbf{g}_{p q}^{\|}, \operatorname{Sign}\left(z_{i}-z_{j}\right) \kappa_{p q}\right) \\
\kappa_{p q} & =\left(k^{2}-\left|\mathbf{k}_{\|}+\mathbf{g}_{p q}^{\|}\right|^{2}\right)^{1 / 2} .
\end{aligned}
$$

The meaning of the symbols, e.g. the surface reciprocal lattice vector $g_{p q}^{\|}$, has been given in ref 2 . Only values of $0^{\circ}$ and $90^{\circ}$ for the anisotropic azimuth angle $\Omega$ will be considered. $\Omega=0^{\circ}$ will be treated in detail, $\Omega=90^{\circ}$ follows by analogy. Since in that case $k_{y}=0$, the $x y$ and $z y$-components of $\overrightarrow{\mathbf{F}}_{i j}$ disappear (then the $p q$-terms in the summation at (6) become antisymmetric). In ref 2 the interaction matrix $M$ was defined through:

$\overleftrightarrow{\mathbf{M}}_{i j}=\overleftrightarrow{\alpha}_{i}^{-1} \delta_{i j}-\alpha_{0}^{-1} \overleftrightarrow{\mathbf{f}}_{i j}$

where $\alpha_{i}$ builds the polarizability tensor, being diagonal for $\mathrm{GaP}$, like $\overleftrightarrow{\mathbf{f}}_{i i}{ }^{1,2}$. So it holds that for $\Omega=0^{\circ}$ for, any $\overleftrightarrow{\mathbf{M}}_{i j}$ the $x z$ component is the only nonzero off-diagonal element. It is not difficult to see that in that case $M$ can be organized such that it becomes blockwise. The first block will contain $y y$-components 
and corresponds to $s$-polarization. The elements of this block are given by (scalar type):

$f_{i i}^{s}=c_{\text {STAT, } y y}+\frac{2 \pi i a^{3} k^{2}}{\left|\mathbf{s}_{1} \times \mathbf{s}_{2} \| k_{\mathbf{z}}\right|}(i=j)$

$f_{i j}^{s}=\frac{2 \pi i a^{3}}{\left|\mathbf{s}_{1} \times \mathbf{s}_{2}\right|} \sum_{p q}^{\infty}\left(k^{2}-g_{p q}^{2}\right) \frac{\exp \left(i \mathbf{k}_{p q} \cdot\left(\mathbf{r}_{i}-\mathbf{r}_{j}\right)\right)}{\kappa_{p q}}(i \neq j)$.

(For $c_{\text {STAT, } y y}$ use 0.9090 .) The solutions of the block $M_{s}$ can be found independently and yield the dipole strength's through:

$p_{i}^{s}=\sum_{j}\left(M_{s}^{-1}\right)_{i j} E_{\mathrm{EXT}, j}^{s}$

The other block containing the $x z$-components and corresponding with $p$-polarization, will be described using $2 d$-vectors/dyads. The elements of this block $M_{p}$ are:

$$
\begin{array}{rlr}
\stackrel{\mathbf{f}}{p}_{i i}^{p} & =\left[c_{\text {STAT, } x x}+\frac{2 \pi i a^{3}}{\left|\mathbf{s}_{1} \times \mathbf{s}_{2}\right|} \frac{\left(k^{2}-k_{x}^{2}\right)}{\left|k_{z}\right|}\right] \hat{\mathbf{x}} \hat{\mathbf{x}} & (12 \mathrm{a}) \\
& +\left[c_{\text {STAT, } z z}+\frac{2 \pi i a^{3}}{\left|\mathbf{s}_{1} \times \mathbf{s}_{2}\right|} \frac{\left(k^{2}-k_{z}^{2}\right)}{\left|k_{z}\right|}\right] \hat{\mathbf{z}} \hat{\mathbf{z}} & (i=j) \\
\overleftrightarrow{\mathbf{f}_{i j}^{p}} & =\frac{2 \pi i a^{3}}{\left|\mathbf{s}_{1} \times \mathbf{s}_{2}\right|} \sum_{p q}^{\infty}\left(k^{2} \overleftrightarrow{1}-\mathbf{k}_{p q}^{p}\left(\mathbf{k}_{p q}^{p}\right)^{T}\right) \frac{\exp \left(i \mathbf{k}_{p q} \cdot\left(\mathbf{r}_{i}-\mathbf{r}_{j}\right)\right)}{\kappa_{p q}}(i \neq j)
\end{array}
$$

$\mathbf{k}_{p q}^{p}=\left(k_{x}+g_{p q, x}^{\|}, \operatorname{Sign}\left(z_{i}-z_{j}\right) \kappa_{p q}\right)$

$c_{\text {STAT, } x x}=4.7901$ and $c_{\text {STAT, } z:}=-5.6961$. Again the solution of the dipole strength's follows independently:

$\mathbf{p}_{i}^{p}=\sum_{j}\left(\stackrel{\leftrightarrow}{\mathbf{M}}_{p}^{-1}\right)_{i j} \mathbf{E}_{\mathrm{EXT}, j}^{p}$

with the $x z 3 d$-components yielding the $2 d$-ones. $\Omega=90^{\circ}$ requires exchange of $x$ - and $y$-directions. Those two anisotropic directions suffice for SIOA and the decomposition reduces the numerical effort drastically.

2.3. Remote fields and ellipsometric parameters. Equations $(17-20)$ of ref 2 for calculation of remote fields still hold. Off normal incidence however requires distinction between $s$ - and $p$-polarization. For any $\mathbf{u}=(x, y, z)$ we define its reflected counterpart $\underline{\mathbf{u}}$ as:

$\underline{\mathbf{u}}=(x, y,-z)$.

Using this convention we can filter out $s$ - and $p$-components by means of:

$r_{u}=\frac{2 \pi i a^{3} k^{2}}{\left|\mathbf{s}_{1} \times \mathbf{s}_{2} \| k_{z}\right|}\left[\frac{\hat{\mathbf{u}} \cdot \mathbf{P}^{R}}{\alpha_{0} E_{0}}\right]$

$t_{u}=(\hat{\mathbf{u}} \cdot \hat{\mathbf{e}})+\frac{2 \pi i a^{3} k^{2}}{\left|\mathbf{s}_{1} \times \mathbf{s}_{2} \| k_{z}\right|}\left[\frac{\hat{\mathbf{u}} \cdot \mathbf{P}^{T}}{\alpha_{0} E_{0}}\right]$

$u=s, p$ and $\hat{\mathbf{e}}$ is the unit vector, $E_{0}$ the strength of the incoming electric field. The quantities $\mathbf{P}^{R}$ and $\mathbf{P}^{T}$ were derived as ref 2:

$$
\begin{aligned}
& \mathbf{P}^{R}=\sum_{i} \exp \left(-i \underline{\mathbf{k}} \cdot \mathbf{r}_{j}\right) \mathbf{p}_{j} \\
& \mathbf{P}^{T}=\sum_{i} \exp \left(-i \mathbf{k} \cdot \mathbf{r}_{j}\right) \mathbf{p}_{j} .
\end{aligned}
$$

The ellipsometric angles $\Psi, \Delta$ follow from the standard expression $^{13.14}$ :

$r_{s} / r_{p}=\tan \Psi \mathrm{e}^{i \Delta}$

In this convention $\Delta$ will run from 0 to $\pi$ by varying the angle of incidence $\vartheta$ from 0 to $\pi / 2$.

\section{Reflection coefficient for semi-infinite crystals}

Equations (24) and hence also (25) from ref 2 can be used unaltered for $\vartheta$ different from 0 . All we need is a different expression for $q_{z}$. We define the quantities $\zeta$ and $\gamma$ as:

$\zeta=\varepsilon_{1}-\sin ^{2} \vartheta$

$\gamma=\left[2\left(\sqrt{\zeta^{2}+\varepsilon_{2}^{2}}-\zeta\right)\right]^{1 / 2} / \varepsilon_{2}$

$\left(\varepsilon_{2} \neq 0\right)$.

Using $\gamma$ the expression for $q_{z}$ becomes:

$q_{z}=-\left[\frac{1}{\gamma}+i \frac{\varepsilon_{2} \gamma}{2}\right] k$

$k$ being the length of $\mathbf{k}$. The actual conversion is done by [2.24]:

$r=\frac{t_{\mathrm{A}} r_{\mathrm{B}}-\exp \left[i q_{z}\left(d_{\mathrm{B}}-d_{\mathrm{A}}\right)\right] t_{\mathrm{B}} r_{\mathrm{A}}}{t_{\mathrm{A}}-\exp \left[i q_{z}\left(d_{\mathrm{B}}-d_{\mathrm{A}}\right)\right] t_{\mathrm{B}}}$.

Labels $A$ and $B$ refer to the two slabs of different thickness. The continuum description enters our model through the bulk dielectric constant $\tilde{\varepsilon}$. Equivalency with the discrete approach is shown in ref 11 .

\section{Numerical results for semi-infinite GaP (110)}

Two photon energies $\hbar \omega$ will be investigated, yielding case I with $\hbar \omega=2.7212 \mathrm{eV}$ and case II with $h \omega=3.6056 \mathrm{eV}$, both being in the region of $\mathrm{GaP} 110$ surface states. Bulk polarizabilities $\alpha_{B}$ and surface polarizabilities $\alpha_{S}$ have been obtained like in ref 2 .

The polarizabilities in units of $\alpha_{0}=\sqrt{2} \pi \varepsilon_{0} a_{c}^{3}=6.38635^{*}$ $10^{-39} \mathrm{Fm}^{2}$ are given in Table 1. For both cases slabs have been investigated with thicknesses varying from 1 to 80 layers. We have calculated the absorbance $A$ by means of:

$A+R+T=1$.

$A$ being the absorbance, $R$ the reflectance and $T$ the transmittance. The absorbance was always positive, as it should be. Only in case of strong absorption slabs show a convergent optical response, for increasing slab thickness. If the absorption is zero or weak, the slabs display the oscillatory response known from transparent films (see ref 13, p 64). Results obtained for slab thicknesses exceeding 12 , depend smoothly from this quantity Results for thin slabs deviate, the smaller the number of layers becomes. This is in good agreement with our earlier conclusion

Table 1. Polarizabilities ( $S$ : Surface, $B$ : Bulk)

\begin{tabular}{lll}
\hline & Case I: $h \omega=2.7212 \mathrm{eV}$ & Case II: $h \omega=3.6056 \mathrm{eV}$ \\
\hline$\alpha_{B}$ & $0.129427+i 3.83507 * 10^{-4}$ & $0.144226+i 6.94431 * 10^{-3}$ \\
$\alpha_{S, x}$ & $0.139011+i 2.66531 * 10^{-3}$ & $0.152692+i 1.49123 * 10^{-2}$ \\
$\alpha_{S . y}$ & $0.133615+i 1.16360^{*} 10^{-3}$ & $0.145023+i 4.53612^{*} 10^{-3}$ \\
$\alpha_{S . z}$ & $0.128262+i 7.26597 * 10^{-4}$ & $0.144225+i 5.01176^{*} 10^{-4}$ \\
\hline
\end{tabular}


(ref 2) about an 'optical surface region' of about 6 layers. The Brewster minimum can be observed in the $p$-polarized reflectance $\boldsymbol{R}_{p}$ for any thickness. Dependency from slab thickness is most prominent in the ellipsometric angle $\Psi$ if the angle of incidence $\vartheta$ is in between the Brewster angle and grazing incidence.

By means of the two slab approach (ref 2) the optical response for semi-infinite GaP (110) has been calculated, which will be discussed now. Figure 1 shows $R_{p}$ for both cases and the two anisotropic azimuth angles $\Omega=0^{\circ}$ and $\Omega=90^{\circ}$. All curves show clearly the Brewster minimum, but on this scale only for case II the anisotropy is strong enough to be seen directly. There the minimum is also clearly different from zero. It is important to note here already, that at the place of the minima there is still anisotropy. In Figure 2 we show the $s$ - and p-polarized reflectance differences obtained for the two cases. With difference results will be meant difference between $\Omega=90^{\circ}$ and $\Omega=0^{\circ}$ data. The s-polarized results are smoothly dropping off for increasing $\vartheta$. Since also the overall $s$-polarized results do not change dramatically in the same range, this direction of polarization is not interesting from the experimental point of view. The zero in $\Delta R_{p}$ can clearly be observed in this figure for both cases.

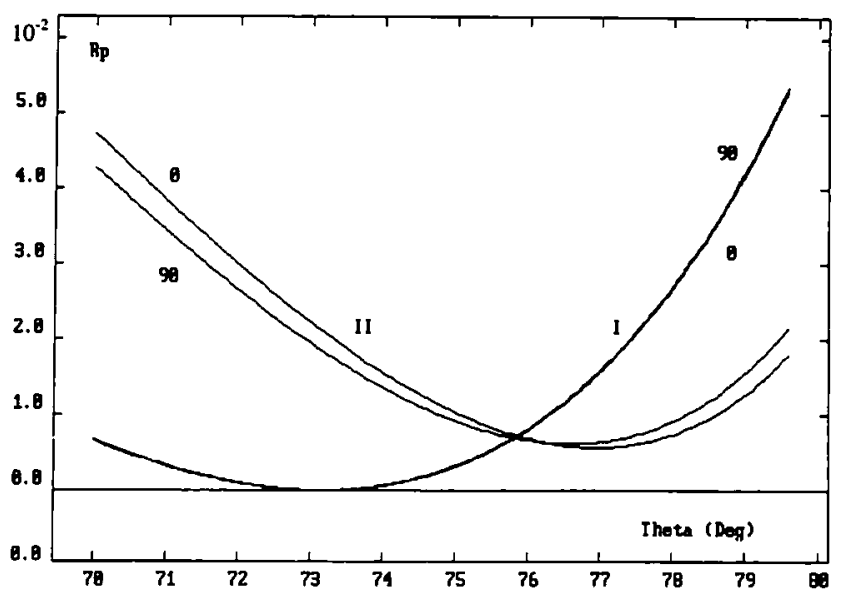

Figure 1. $R_{p}$ and $R_{s}$ for $\Omega=0^{\circ}$ and $\Omega=90^{\circ}$ as a function of $\vartheta$ for case I and case II (two slab approach).

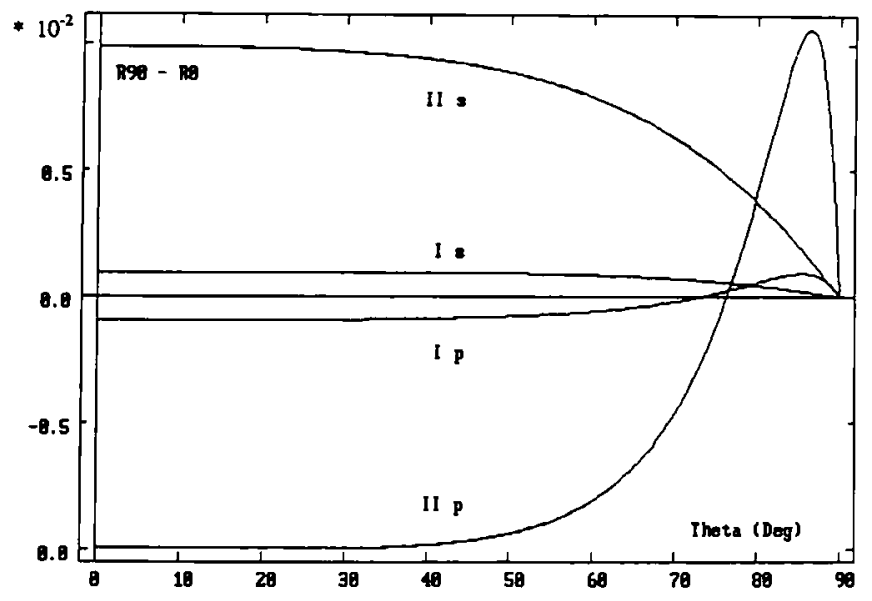

Figure 2. $\Delta R_{p}$ and $\Delta R_{s}$ as a function of $\vartheta$ for case I and case II (two slab approach).

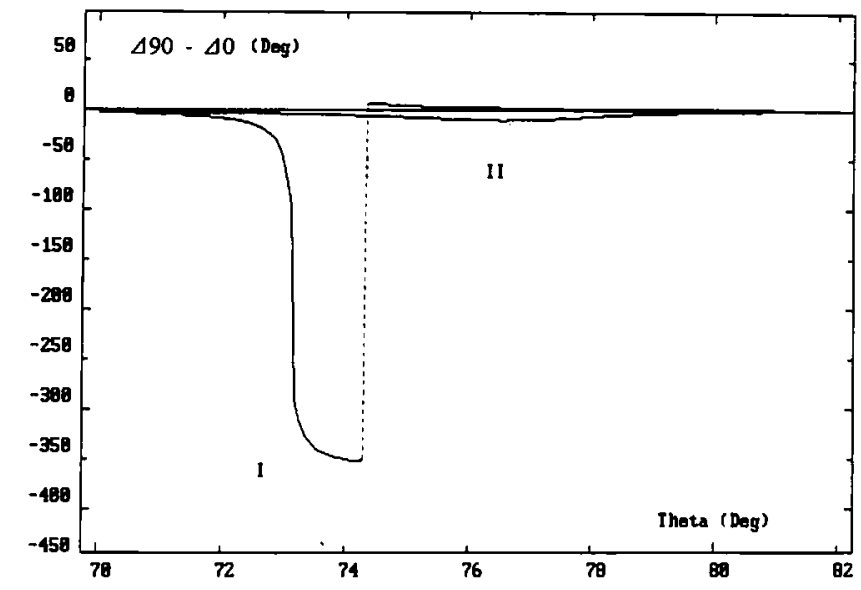

Figure 3. $\delta \Delta=\Delta 90-\Delta 0$ as a function of 9 for case I and case II (two slab approach).

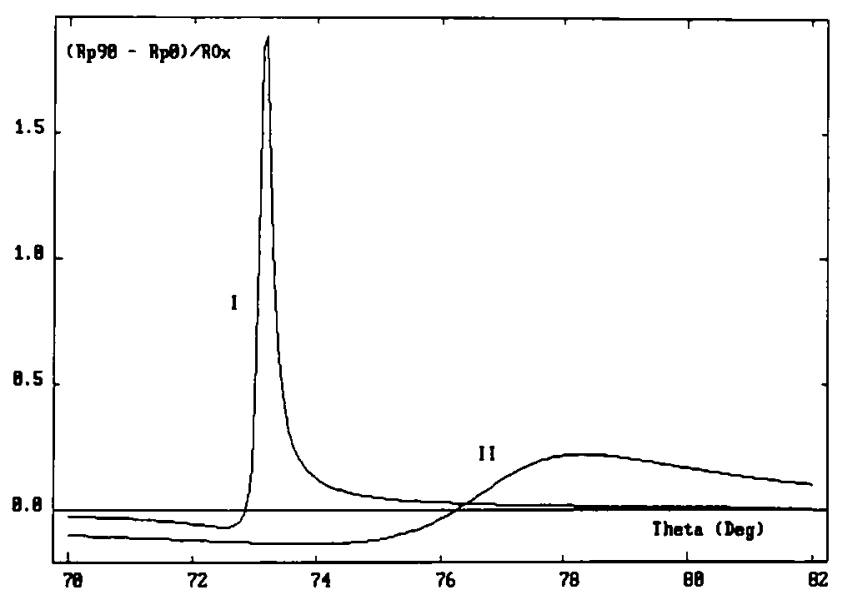

Figure 4. $\Delta R_{p} / \bar{R}_{p}$ as a function of $\vartheta$ for case I and case II (two slab approach).

This phenomenon takes place just before the Brewster minima will be attained. Since the angle $\Psi$ is less sensitive for anisotropy than $\Delta$, we discuss only the latter. Figure 3 shows the ellipsometric difference angle $\delta \Delta$. This difference angle clearly demonstrates that near the Brewster minimum strong effects can be expected. For case I a full sweep of $360^{\circ}$ has been found. The sudden jump upward at $\vartheta=74.5^{\circ}$ however has no physical meaning, since one can always add a multiple of $360^{\circ}$. Case II displays a more moderate behaviour, having a minimum of $-10.3^{\circ}$ for $\vartheta=76.5^{\circ}$ and a half width of $4.6^{\circ}$. The sensitivity of $\Delta$ is obviously due to the contribution of $r_{p}$ to (18). This has been investigated separately by looking at the figure of merit $\Delta R_{p} / \bar{R}_{p}$, shown in Figure 4. The higher this figure of merit, the higher will be the theoretically possible signal to noise ratio. Since $\bar{R}_{p}$ has been defined as $0.5^{*}\left(R_{p} 90+R_{p} 0\right)$ and $R_{p}$ by definition is positive, $\Delta R_{p} / \bar{R}_{p}$ will not exceed 2.0. This value is almost the maximum for case 1 , being 1.8. Case II produces a much smaller maximum of 0.22 for $\vartheta=78.4^{\circ}$. This is however still a factor of 8.5 better than for perpendicular incidence. These theoretical values can only be obtained in experiment, if the reproducibility of the angle of incidence is close to perfect. In that sense case II represents the easier frequency. Despite those problems the higher sensitivity 
for SIOA will be found according to this model near Brewster's minimum and especially the optical observation of surface state related phenomena at subbandgap conditions may benefit from that.

\section{Conclusions and remarks}

In this article has been shown how variation of the angle $\vartheta$ influences surface induced optical anisotropy (SIOA). The angular dependent total response for both slabs and semi-infinite bulk has been discussed. As to the latter the hybrid technique (ref 2), used before to calculate semi-infinite results, turned out to be equally well usable. Rather against expectation, the $p$ - and not the $s$-polarization turned out to be the experimentally more sensitive direction. Especially for low absorption, as is the case at subbandgap conditions, this sensitivity becomes apparent in $\delta \Delta$ near the Brewster angle, but the experimentally preferable quantity should be $\Delta R_{p}$ at the same angle. As a final remark it should be kept in mind that the theoretical approach used for this article predicts very well the overall response, but produces too high values for the difference results (ref 2). However we think that the main conclusions of this article will not be affected by this comment.

\section{References}

${ }^{1}$ C M J Wijers and K M E Emmett, Physica Scripta, 38, 435 (1988).

${ }^{2}$ C M J Wijers and R Del Sole, Physica Scripta, T25, 325 (1989).

${ }^{3}$ V L Berkovits, I V Makarenko, T A Minashvili and V I Safarov, Solid State Commun, 56, 449 (1985).

${ }^{4} \mathrm{~V}$ L Berkovits, L F Ivantsov, I V Makarenko, T A Minashvili and V I Safarov, Solid State Commun, 64, 767 (1987).

${ }^{5} \mathrm{R}$ Del Sole and E Fiorino, Phys Rev, B29, 4631 (1984).

${ }^{6}$ A Bagchi, R G Barrera and A K Rajagopal, Phys Rev, B20, 4824 (1979).

7 W L Mochán and R G Barrera, Phys Rev Lett, 55, 1192 (1985).

${ }^{8}$ P P Ewald, Ann Phys, 49, 117 (1916); 54, 519 and 557 (1917).

9 O. Litzman, Optica Acta, 27, 231 (1980).

${ }^{10} \mathrm{O}$ Litzman and P Rózsa, Surface Sci, 66, 542 (1977).

1 G P M Poppe and C M J Wijers, Proc 10th Colloq Hertzian Optics and Dielectrics, Rennes (1989).

${ }_{12} \mathrm{C}$ M J Wijers, R Del Sole and F Manghi, Phys Rev B, to be published. ${ }^{13} \mathrm{M}$ Born and E Wolf, Principles of Optics. Pergamon Press, Oxford (1985). 